\title{
Self-Reconfigurable Modular Robots Adaptively Transforming a Mechanical Structure: Algorithm for Adaptive Transformation to Load Condition
}

\author{
Yosuke Suzuki, ${ }^{1}$ Norio Inou, ${ }^{2}$ Hitoshi Kimura, ${ }^{2}$ and Michihiko Koseki ${ }^{3}$ \\ ${ }^{1}$ Graduate School of Informatics and Engineering, The University of Electro-Communications, 1828585 Tokyo, Japan \\ ${ }^{2}$ Department of Mechanical and Control Engineering, Tokyo Institute of Technology, 1528550 Tokyo, Japan \\ ${ }^{3}$ Faculty of Textile Science and Technology, Shinshu University, 3868567 Nagano, Japan
}

Correspondence should be addressed to Yosuke Suzuki, suzuki@mce.uec.ac.jp

Received 14 July 2011; Accepted 25 October 2011

Academic Editor: Gordon R. Pennock

Copyright ( 2011 Yosuke Suzuki et al. This is an open access article distributed under the Creative Commons Attribution License, which permits unrestricted use, distribution, and reproduction in any medium, provided the original work is properly cited.

\begin{abstract}
Self-reconfigurable modular robots are composed of modules which are able to autonomously change the way they are connected. An appropriate control algorithm enables the modular robots to change their shape in order to adapt to their immediate environment. In this paper, we propose an algorithm for adaptive transformation to load condition of the modular robots. The algorithm is based on a simple idea that modules have tendency to gather around stress-concentrated parts and reinforce the parts. As a result of the self-reconfiguration rule, the modular robots form an appropriate structure to stand for the load condition. Applying the algorithm to our modular robot named "CHOBIE II," we show by computer simulation that the modules are able to construct a cantilever structure with avoiding overstressed states.
\end{abstract}

\section{Introduction}

A robot system which has an ability to reorganize its own mechanism would be singularly able to fulfill various tasks, by switching its locomotion mechanism according to the immediate terrain and by changing its end-effectors for manipulating objects. Modular robots, which are composed of multiple autonomous modules, are able to self-reconfigure by replacing the geometric arrangement of the modules forming the robot system. This paper focuses on "load adaptation" by self-reconfiguration. The novel function would enable modular robots to autonomously change their configuration to the most appropriate one for enduring even unpredictable load condition, as if the robots were body tissue of natural life forms. A variety of mechanisms of modular robots have been developed [1-13]. Chain-type robots such as PolyBot [4] and CONRO [7] place an emphasis on mobility of the multidegree-of-freedom systems, and lattice-type robots such as Crystalline [5] and ATRON [8] mainly focus on geometrical transformation of the modular structures. However, there are very few designs for supporting large external forces, and an algorithm for load-adaptive transformation has never discussed. It is because the almost developed modular robots are designed for the mobile or transformation function than the supporting function.

From such a point of view, we have developed modular robots named "CHOBIE II" forming a mechanical structure which has adaptability to a load condition [14-16]. CHOBIE II has the following properties.

(1) CHOBIE II consists of identical modules in block-like shape as shown in Figure 1.

(2) The modules construct a 2D lattice structure.

(3) The structural transformation is performed by cooperative slide movements of the modules (like a sliding block puzzle).

(4) Each module determines its own action by the internal processor communicating with adjacent modules.

(5) The same control program is installed in all modules. 


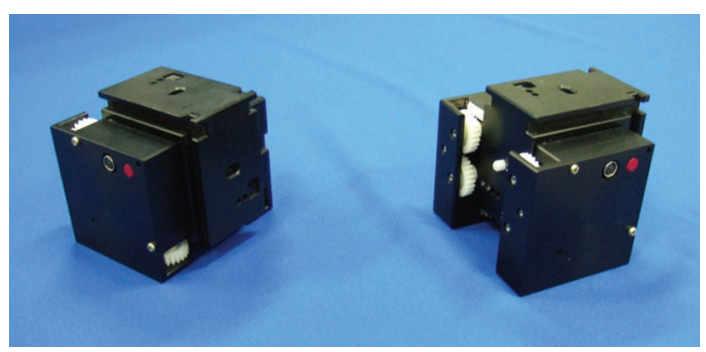

FIGURE 1: External view of the modules of CHOBIE II.

In the previous research $[15,16]$, we proposed a method to input criteria for generating transformations within a framework of a decentralized system. The method focuses on undesirability of contour shape of the structure, and the criterion is regulated by 32 control parameters which correspond to 32 kinds of local states of the contour shape. According to the criterion, the modules can dissolve undesirable local shapes and eventually transform to a goal configuration, even though all of the modules act according to the same control program. The simplicity and versatility are similar to the hormone-inspired scheme by Shen et al. [7]. We have realized structural transformation to various goal configurations by the actual hardware, however, we have not yet examined adaptability to the load condition. The adaptability is an important function for structural formation because robot structures are often exerted by loading force especially on arms, legs, end-effectors, and other functional parts.

This paper discusses a method to realize load adaptive motion by adding new criteria. Preparatory to the discussion, the mechanism and algorithms using previous criterion are described in Sections 3 and 4. Then, in Section 5, we propose two additional criteria, and enable CHOBIE II to construct a bridge-like structure well balanced in stress distribution as shown in Figure 2. One is a strategy to induce desirable local shape, which enables CHOBIE II to transform to characteristic configurations like a bridge. The other is a rule that the modules convert the control parameters for motion generation depending on local load conditions. We demonstrate that, although each module only processes distributed information, the whole modules can transform to a bridge-like structure with reinforcing stressconcentrated portions. Since the algorithm proposed here is based on a very simple methodology that overloaded states are undesirable for the structure, so it would be also available for modular robots other than CHOBIE II.

\section{Related Works}

Few researches have approached adaptive transformation to mechanical load condition of modular robots. One approach was presented by Bojinov et. al [13]. They proposed a control algorithm that modules forming a table-like structure generate a new leg or remove an extra leg according to the load distribution on the table top, and they showed by simulations that the arrangement of the table legs became appropriate for enduring the given load condition. However, since this algorithm enables only generation or removal of legs, it cannot work in shapes other than the table or in complicated load conditions such as including external force pointing in different directions. In addition, designed only for load adaptive transformation of all-ready constructed table, this algorithm cannot achieve both shape construction and load adaptation in the same control rule.

In consideration of the algorithm, this paper proposes a new algorithm with the following advantages.

(1) The algorithm does not depend on the shape or load condition of the structure because it is based on a simple methodology that reinforcement of the structure can be achieved by moving a module to the stress-concentrated portion.

(2) The control rules for load adaptation can be additionally introduced in the shape construction algorithm, and so the modular robot can perform load adaptation only when necessary during working for the main purpose of shape construction.

Our algorithm is inspired by remodeling function of bone tissue, where resorption of old tissue and formation of new tissue occur as metabolism of the bone, and both processes are always kept at equilibrium state [17-20]. When the environment around the bone changes and the equilibrium is disturbed, the shape or property of the bone also changes so as to transit at new equilibrium state. For example, when external force acts on a bone, formation of new tissue is excited and, as a result, the skeleton becomes thick and rigid, whose shape is more adapted for enduring the load condition than before loading. Therefore, the remodeling function enables adaptive variations of living tissue according to its varying environment. We try to apply this mechanism to the reconfiguration algorithm of modular robots. In our new algorithm, each module detects the stress condition and varies its criterion for generating transformation of the structure according to the magnitude of stress. This method enables the modules to keep to an appropriate configuration against their immediate load condition, while each of the modules decides its own action in a distributed autonomous control manner.

Another merit of the algorithm for load adaptation is facility of combination with algorithms for structural construction. As the operation shown in Figure 2, the primary purpose of the modular robots is to construct an objective configuration like a bridge, and load adaptive transformation is just a secondary purpose only required if large stress occurs in the structure. So, an important issue is that load adaptive transformation must not disturb the primary structural construction. To reflect the demand, the two algorithms should be combined with consistency. In this paper, we propose an algorithm in which motivation for load adaptation can be included in the transformation rule for structural construction. We show that the algorithm realizes adaptive transformations appropriate for the objective construction. 


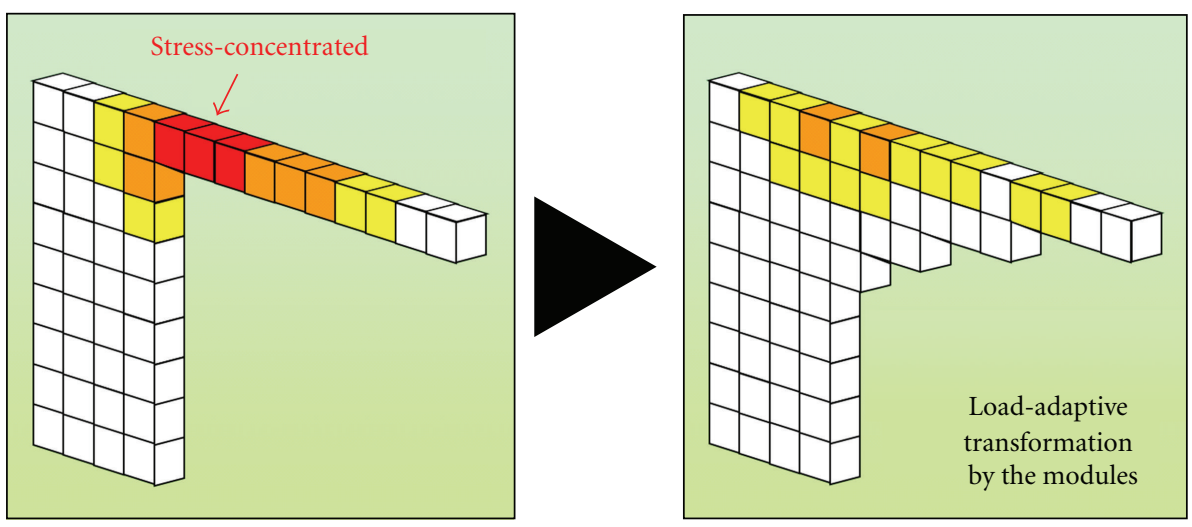

FIGURE 2: Objective structural transformation (modules construct a cantilever structure with reinforcing the overstressed parts).

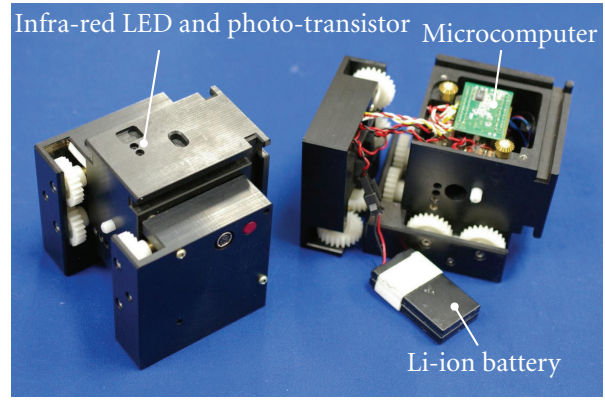

(a)

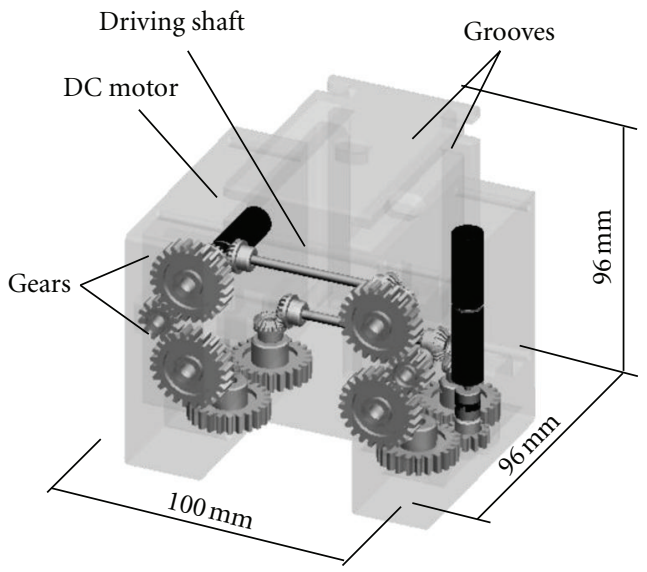

(b)

FIGURE 3: Mechanism of the modules of CHOBIE II (a) outside/inside views of the module, (b) slide motion mechanism.

\section{Mechanism of CHOBIE II}

We describe the mechanisms of the CHOBIE II module. Figure 3 shows the overview and the proposed slide motion mechanism of CHOBIE II system. It consists of two lateral boards and a central board. The two lateral boards include symmetrical motion mechanisms that consist of two sets of gears as shown in Figure 3(b). They are allocated in vertical and horizontal directions, which enable the two directional movements of modules. On the other hand, the central board has grooves as sliding guides as shown in Figure 4(a). Although the degree of freedom is less than that of Crystalline which also applies sliding transformation [4], the mechanism of CHOBIE II maintains high rigidity even during transformation.

These mechanisms enable adjacent modules to keep joining each other strictly. In addition, since the block-like shape of the module has high space-filling property, CHOBIE II constructs a sturdy 2 dimensional lattice structure. Transformations of the structure are carried out by simultaneous slide movements of modules in a straight line, that is, a specific "row" or "column" as shown in Figure 4(b).
The communication devices between neighboring modules are composed of infrared LEDs and phototransistors. One LED and one phototransistor are set on each of the four contact surfaces, and they are allocated so that an LED of a module faces to a phototransistor of another module when the two modules are neighboring. By turning ON/OFF the LEDs, the modules send signals to neighboring modules. Furthermore, strain gauges are used as force sensors to obtain load conditions of the modules. The gauges are attached at the weakest position in the module structure, where the largest stress occurs, as shown in Figure 5. The specifications of the module are shown in Table 1.

\section{Transformation Algorithm}

To generate synchronous slide movements of the CHOBIE II modules within a framework of the decentralized system, we have proposed "temporary leader scheme" where the modules elect their leader in each transformation turn. In this scheme, each module calculates motivation to become a leader based on the local shape around the module and pregiven 32 control parameters corresponding to undesirability of local shapes. The procedure is as follows. 

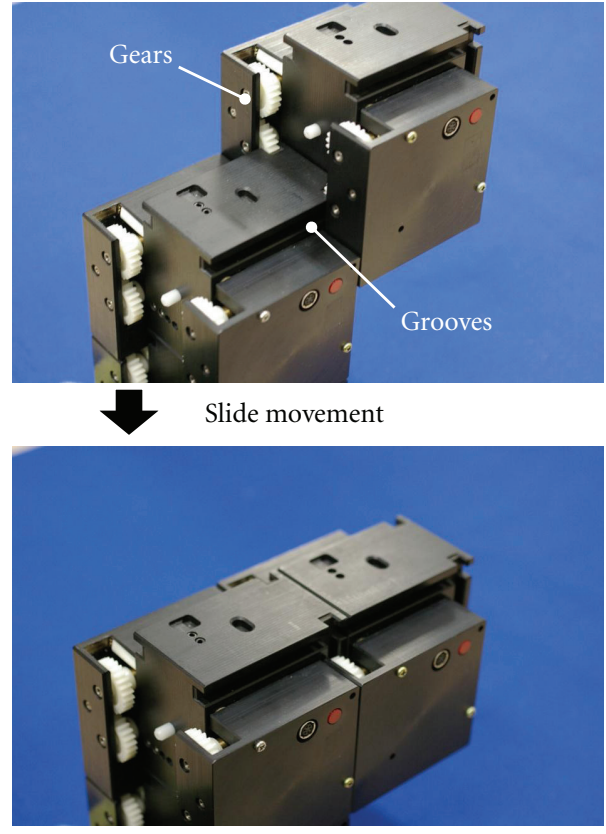

(a)
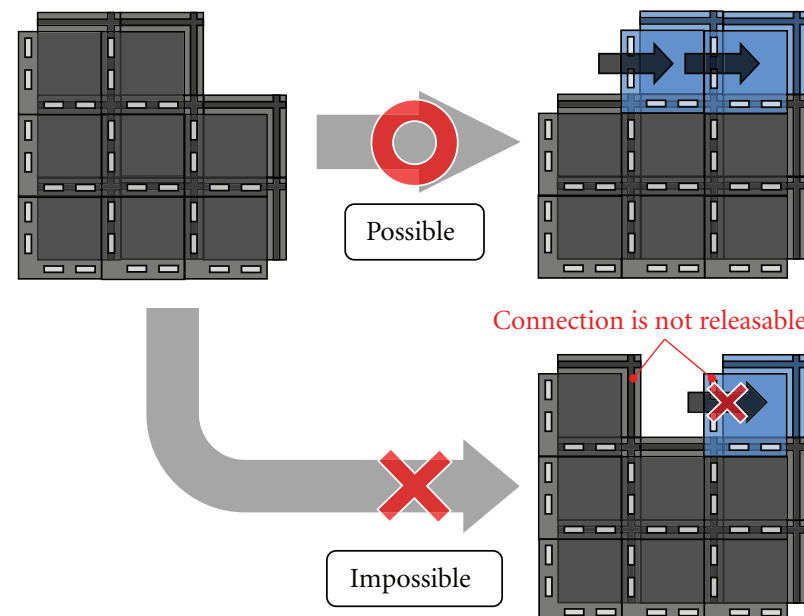

Connection is not releasable

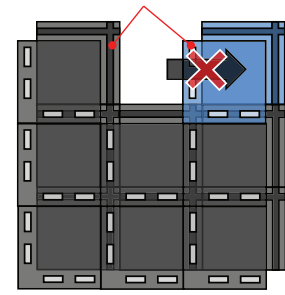

(b)

Figure 4: Structural transformation of CHOBIE II (a) connection of the modules by slide motion, (b) mechanical constraint in transformation.

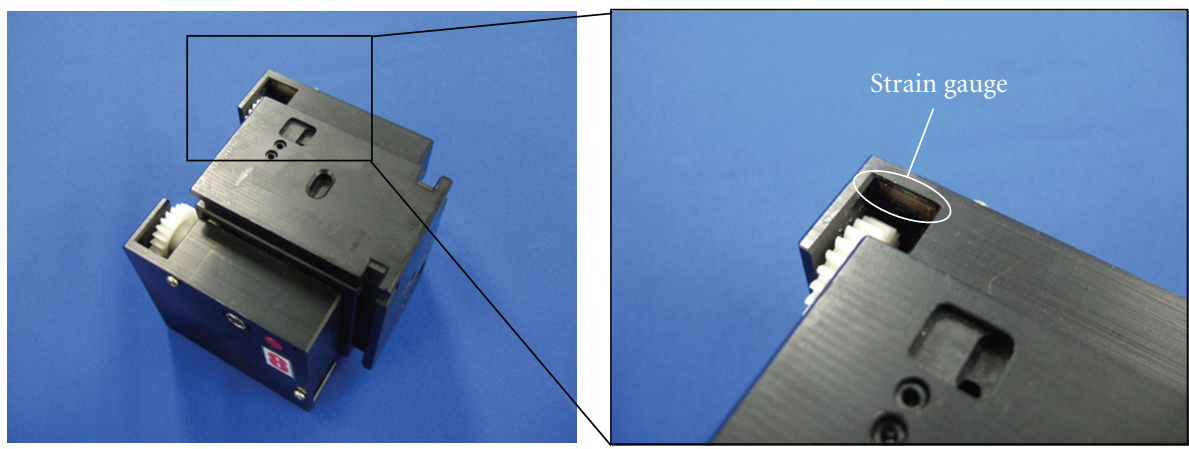

FIGURE 5: Position of strain gauge sensor.

TABLE 1: Specification of the modules of CHOBIE II.

\begin{tabular}{ll}
\hline Main material & ABS resin \\
\hline \multirow{3}{*}{ Size } & Central board: $80 \times 80 \times 50[\mathrm{~mm}]$ \\
& Lateral board: $80 \times 80 \times 25[\mathrm{~mm}]$ \\
Mass & Outer size: $96 \times 96 \times 100[\mathrm{~mm}]$ \\
Driving force & $0.59[\mathrm{~kg}]$ \\
& $20[\mathrm{~N}]$ \\
Moving velocity & Upward: $44.4[\mathrm{~mm} / \mathrm{s}]$ \\
& Downward: $57.1[\mathrm{~mm} / \mathrm{s}]$ \\
MPU & Sideways: $53.3[\mathrm{~mm} / \mathrm{s}]$ \\
Battery & H8/3664F \\
\hline
\end{tabular}

(1) Each module obtains information about 8 kinds of local characteristic shapes on both ends of its row and column. We call these shapes Cv1 Cv4 and Ch1 Ch4.

Cv1: Upper end of the column is left end Cv2: Upper end of the column is right end Cv3: Lower end of the column is left end Cv4: Lower end of the column is right end Ch1: Left end of the row is upper end Ch2: Left end of the row is lower end Ch3: Right end of the row is upper end Ch4: Right end of the row is lower end.

Then, each module substitutes 0 or 1 into binary vectors $\mathbf{c}_{\mathbf{v}}=$ $\left\{\begin{array}{llll}c_{v 1} & c_{v 2} & c_{v 3} & c_{v 4}\end{array}\right\}^{T}$ and $\mathbf{c}_{\mathbf{h}}=\left\{\begin{array}{llll}c_{h 1} & c_{h 2} & c_{h 3} & c_{h 4}\end{array}\right\}^{T}$ according to the existence of the local characteristic shapes as an example shown in Figure 6. 


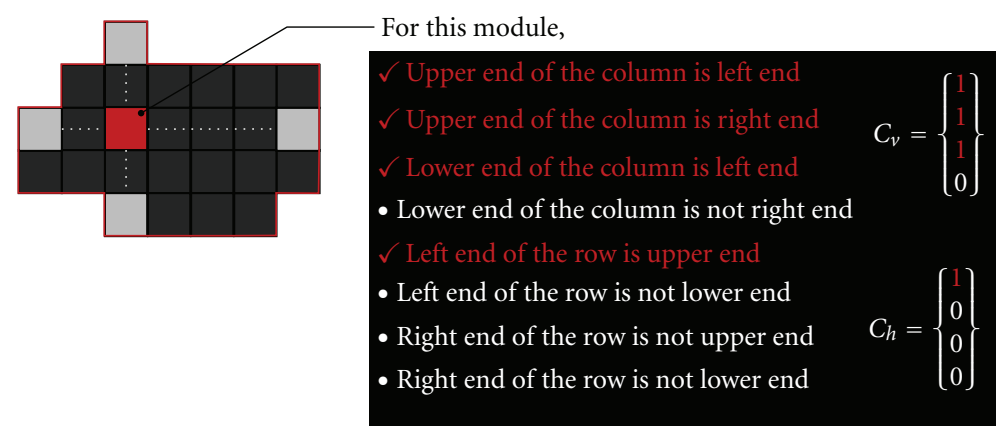

FIgURE 6: An example of concerned local shapes about a module.

(2) About each of the 8 shapes, combined states with other 4 shapes, that is, totally 32 kinds of state are considered as shown in Figure 7. We give undesirability of the states by the following 32 parameters:

$$
\begin{gathered}
A_{v}=\left[\begin{array}{llll}
a_{v 11} & a_{v 12} & a_{v 13} & a_{v 14} \\
a_{v 21} & a_{v 22} & a_{v 23} & a_{v 24} \\
a_{v 31} & a_{v 32} & a_{v 33} & a_{v 34} \\
a_{v 41} & a_{v 42} & a_{v 43} & a_{v 44}
\end{array}\right], \\
A_{h}=\left[\begin{array}{llll}
a_{h 11} & a_{h 12} & a_{h 13} & a_{h 14} \\
a_{h 21} & a_{h 22} & a_{h 23} & a_{h 24} \\
a_{h 31} & a_{h 32} & a_{h 33} & a_{h 34} \\
a_{h 41} & a_{h 42} & a_{h 43} & a_{h 44}
\end{array}\right] .
\end{gathered}
$$

Here, placement of the parameters corresponds to that of the 32 states in Figure 7.

(3) If there are some of the 32 states around a module, the module refers to the corresponding parameters. Then, each module calculates summation of the parameters $L$ as follows. The value $L$ is called "leader index":

$$
L=c_{v}^{T} A_{v} c_{h}+c_{h}^{T} A_{h} c_{v}
$$

(4) A module which has the largest leader index becomes a temporary leader.

(5) The leader module sends a drive command to dissolve the most undesirable characteristic shape around the leader.

(6) Modules perform one grid of slide movement following the drive command.

In this method, although each module deals with only local information on its row and column in a decentralized manner, the whole structure can generate transformation for dissolving undesirable shapes. The criterion is regulated by 32 parameters $A_{v}$ and $A_{h}$ in (1) which mean undesirability of local contour shapes in Figure 7. By setting these parameters appropriately, we can enable CHOBIE II to perform various motions.

As a simple example, we show a case giving parameters as

$$
A_{v}=\left[\begin{array}{llll}
0 & 0 & 0 & 0 \\
0 & 0 & 0 & 0 \\
0 & 0 & 0 & 0 \\
0 & 0 & 0 & 0
\end{array}\right], \quad A_{h}=\left[\begin{array}{llll}
0 & 0 & 0 & 0 \\
0 & 0 & 0 & 0 \\
0 & 1 & 0 & 0 \\
0 & 0 & 0 & 0
\end{array}\right] .
$$

In these parameters, only $\alpha_{h 32}$ has a value, which means, in a condition that "upper end of the column is right end" and "right end of the row is upper end," then the shape on right end of the row is undesirable. Consequently, a module satisfying the condition becomes a leader. Then, in order to dissolve the undesirable shape, the leader commands modules on the upper row slide to rightward as shown in Figure 8 . By repeating this process, CHOBIE II transforms to a configuration without undesirable local shapes.

Figure 9 shows a simulation result of this motion. In this figure, red color in inner parts of modules becomes bright in proportion to leader indexes of the modules, and a white "L" mark means a leader module. The number of leader in each transformation is basically one, however, when two or more modules are in the same condition, the modules may become leaders at the same time.

The most important feature of the motion-generating method is that a module which becomes a leader is not always a module which has the most undesirable local shape, but a module which has the largest value assessed about all shapes around the module. Therefore, complicated transformation process can be generated by adjusting magnitude relations of the parameters in an appropriate manner.

However, the above criteria only give a strategy to dissolve undesirable local shapes. We need a new strategy to make CHOBIE II construct a characteristic structure like a bridge. It is possible to solve the problem by extending the criteria expressed by 32 parameters.

\section{Load Adaptation Algorithm}

The algorithm for structural construction described in Section 4 adopts rules for generating transformations only to 

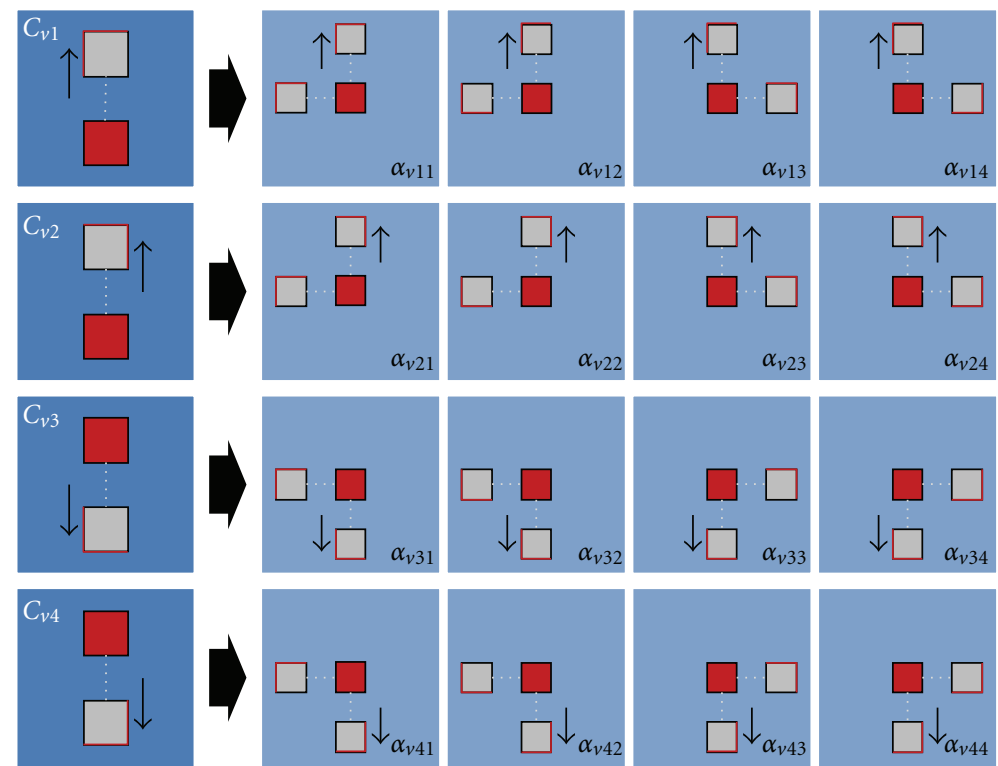

(a)
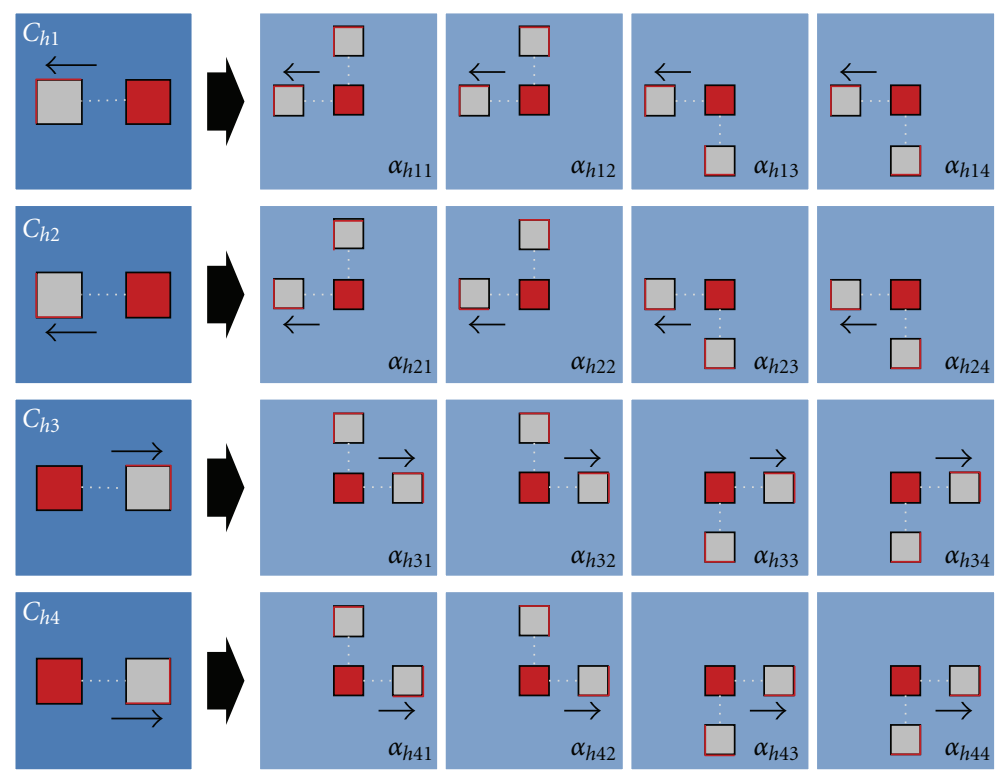

(b)

FIGURE 7: 32 patterns of states which are set "undesirability" parameters (undesirability of each of the 8 kinds of characteristic shapes is given by 4 parameters).

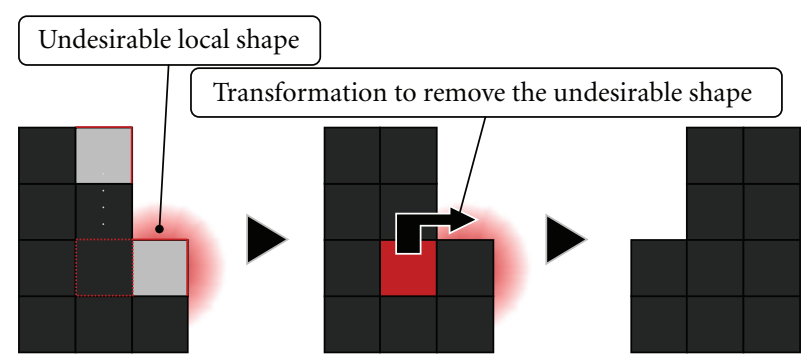

Figure 8: Methodology to generate transformations based on undesirability of local shapes. dissolve undesirable local shapes. To realize the motion that CHOBIE II constructs a bridge-like structure with adapting to its load condition as shown in Figure 2, two new rules must be added to the above algorithm. First, we add a strategy to induce desirable local shapes and enable CHOBIE II to transform to characteristic configurations. Next, we realize load adaptive motion of CHOBIE II by setting a rule that the modules give more attention to local shapes in the direction where large stress occurs.

5.1. Inducement of Desirable Local Shapes. First, we explain expression of desirable states. As described in Section 4, the 

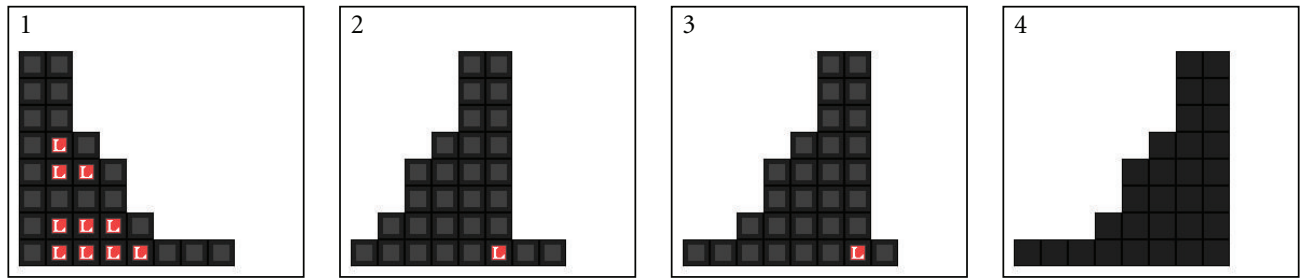

FIGURE 9: Simulation result of transformation process.

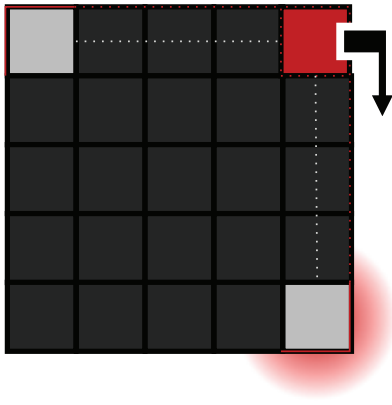

(a) $a_{v 41}>0$

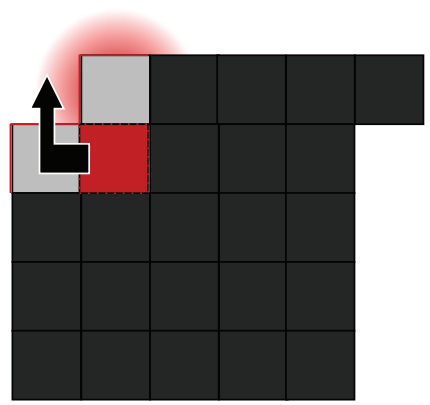

(b) $a_{v 11}>0$

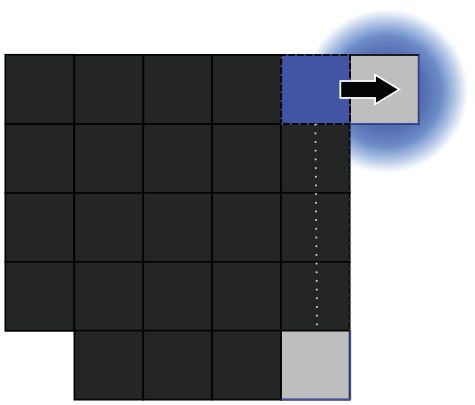

(c) $a_{h 44}>0$

Figure 10: Transformation modes for cantilever construction.

32 parameters for regulating a criterion are corresponding to the 32 kinds of states. When a parameter is 0 , the corresponding state is not undesirable. As the parameter becomes larger, the state is considered to be more undesirable. That is, by inputting a parameter lower than 0 , we can express that the corresponding state is desirable.

In this regard, different patterns of transformations should be generated according to whether the corresponding state is undesirable or desirable. Therefore, the procedure has a difference from that of Section 4 . After calculating leader index in the same way as (1), (2), and (3), then, at (4), a module which has the largest "absolute value" of leader index becomes a leader. If the leader index of the leader is lower than 0 , the drive command from the leader at (5) changes to the direction for inducing the desirable shape, that is, opposite to the direction in the case of undesirable shape.

Applying this method, we perform a simulation of constructing a cantilever structure from a rectangle structure. There are three transformation modes required for this motion, as shown in Figures 10(a), 10(b), and 10(c).

The mode (a) is for generating rightward slide movements on top row of the rectangle structure at the first step, and the mode (b) is for supplying modules to the upper side which become construction elements of the cantilever. Then, the mode (c) is for lengthening the cantilever. Among the 32 parameters, $a_{v 41}, a_{v 11}, a_{h 44}$ are related to each of the modes. Here, the former two modes are dissolution of undesirable shapes, and the latter mode is inducement of a desirable shape. Therefore, these parameters have to satisfy conditions, $a_{v 41}>0, a_{v 11}>0, a_{h 44}<0$. In addition, priorities of the modes are in the order of (b) $>$ (c) $>$ (a). From these points, the parameters are set so that $\left|a_{v 41}\right|>\left|a_{h 44}\right|>\left|a_{v 11}\right|$, and all the other parameters are 0 .

Figure 11 shows a simulation result in the setting of following formula:

$$
A_{v}=\left[\begin{array}{llll}
3 & 0 & 0 & 0 \\
0 & 0 & 0 & 0 \\
0 & 0 & 0 & 0 \\
1 & 0 & 0 & 0
\end{array}\right], \quad A_{h}=\left[\begin{array}{cccc}
0 & 0 & 0 & 0 \\
0 & 0 & 0 & 0 \\
0 & 1 & 0 & 0 \\
0 & 0 & 0 & -2
\end{array}\right] .
$$

The simulation shows that the modules gradually construct a cantilever structure. From this result, it is expected that CHOBIE II can construct various distinctive structures by using this method.

5.2. Implementation of Adaptability. Next, we introduce a method to realize load adaptive motions considering stressed states of the modules in order. For example, when the foregoing cantilever structure (Figure 11) is actually formed in the gravity field, overload caused by the weight of the modules themselves may damage some modules. To avoid the problem, it is necessary that the modules can construct a cantilever with reinforcing overstressed parts which occur in the construction process. For such an adaptive motion, the criterion for generating transformation has to change according to whether there are overstressed modules in the structure or not. 

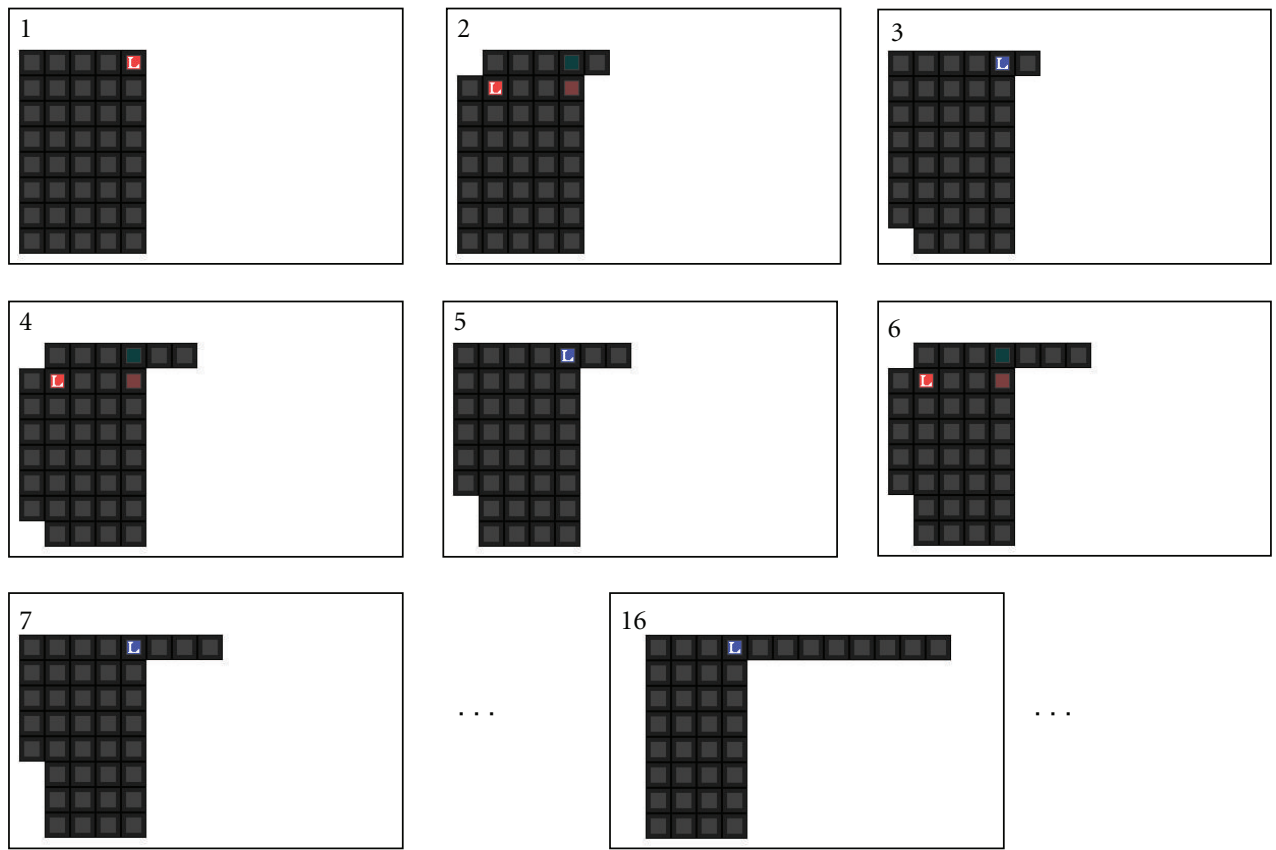

FIgURE 11: Simulation result of cantilever construction from $8 \times 5$ rectangle structure.

Over-stressed module

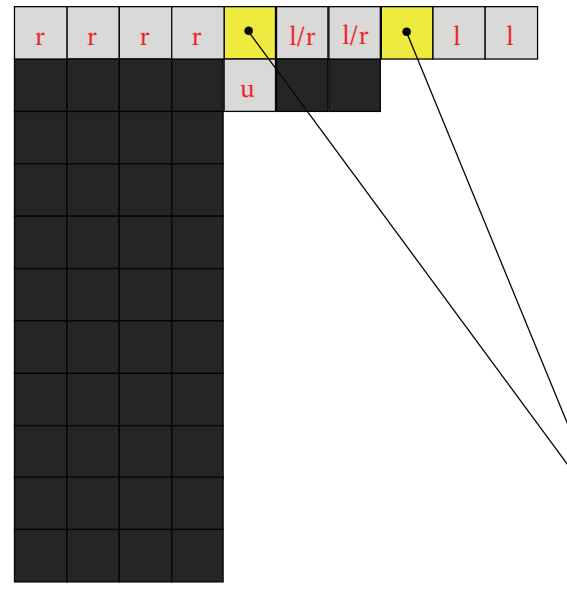

- The "r" marked modules evaluate undesirability/desirability of the shapes in right direction with multiplying certain gain

- The "l" marked modules evaluate undesirability/desirability of the shapes in left direction with multiplying certain gain

- The " $1 / \mathrm{r}$ " modules evaluate

undesirability/desirability of the shapes in both left and right direction with multiplying certain gain

- The "u" module evaluates

undesirability/desirability of the shapes in upper direction with multiplying certain gain

The overstressed modules evaluate

undesirability/desirability in ordinary way (without multiplying the again)

FIGURE 12: Alternation of the evaluation rule of undesirability/desirability of the shapes when there are overstressed modules in the structure.

For the purpose, we add the following rules. When a module calculates its leader index, if there are overstressed modules in some directions on the row or column, parameters corresponding to the shapes in the directions are multiplied by a certain gain. Exceptionally, the overstressed modules calculate their leader indexes without multiplying the gain. An example case is shown in Figure 12.

Here, two values of gain are used depending on the situation; a gain more than 1 is multiplied if a shape is undesirable (the corresponding parameter is more than 0 ), and a gain less than 1 is multiplied if a shape is desirable (the corresponding parameter is less than 0 ). We call the former gain $G^{+}$, and the latter $G^{-}$. This rule makes the modules estimate more undesirably about shapes which cause overstressed states, and transformations to dissolve such shapes become to be generated with higher priority. That is, we can set that a shape is not so undesirable in normal situations but is very undesirable in critical situations. Therefore, it is possible to shift the priorities of the transformation modes according to existence of overstressed states. The existence of the overstressed states can be detected by decentralized signal transfer from the overstressed modules.

The parameters for constructing a cantilever structure with adapting to a load condition are determined as follows. 


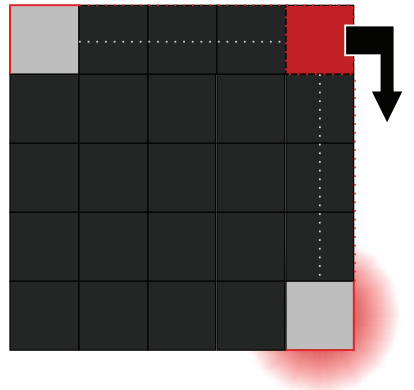

(a) $a_{v 41}>0$

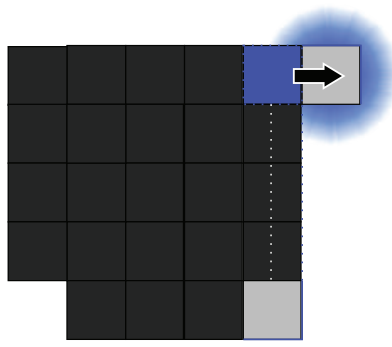

(d) $a_{h 34}<0$

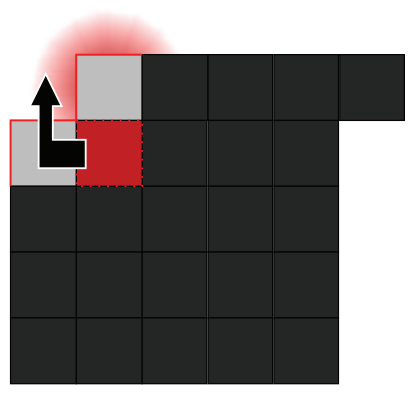

(b) $a_{v 11}>0$

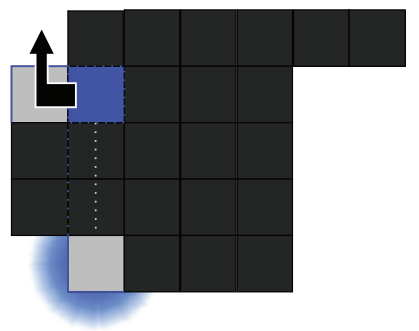

(e) $a_{v 31}<0$

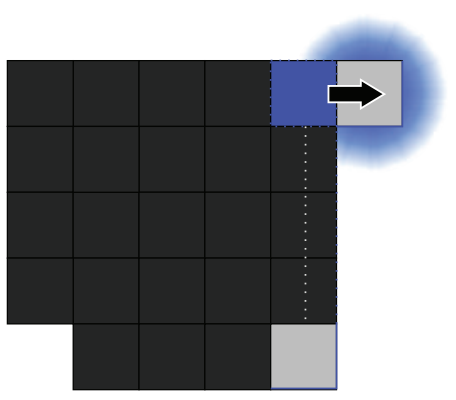

(c) $a_{h 44}<0$

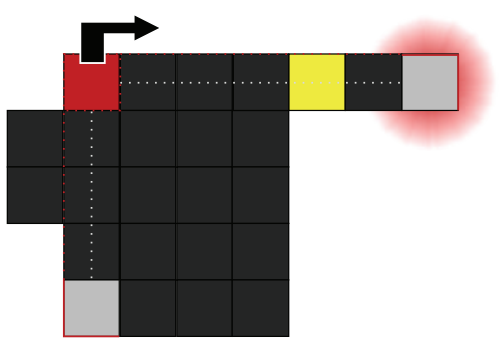

(f) $a_{h 33}>0$

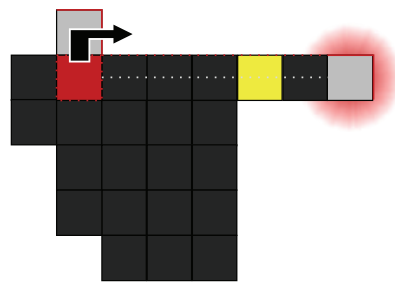

(g) $a_{h 32}>0$

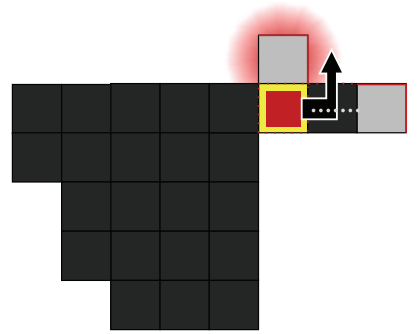

(h) $a_{h 23}>0$

FIGURE 13: Transformation modes for adaptive cantilever construction.

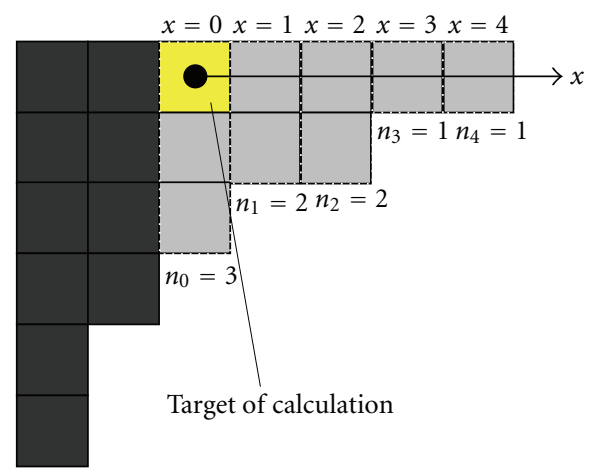

(a)

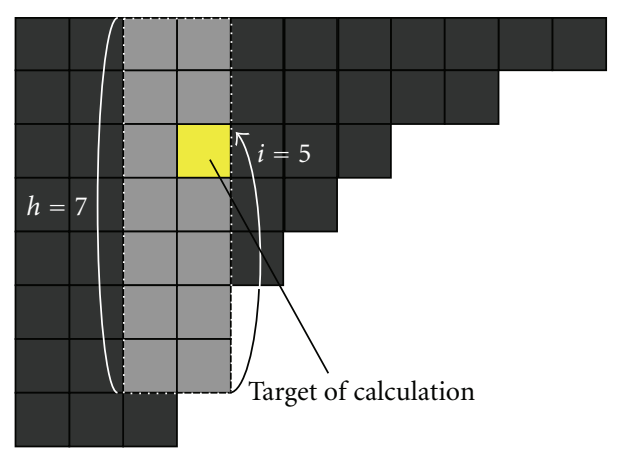

(b)

FIGURE 14: Estimation of strain gauge output.

Figure 13 shows eight transformation modes required for the motion. The modes (a), (b), and (c) are for constructing cantilever as described in the previous section. In this motion, the mode (d) is additionally used for constructing the cantilever. When the cantilever gets long and overstressed states occur in modules denoted by yellow-colored squares, on the effects of modes (b), (e), and (f), a module for reinforcement is transported above the overstressed module.
Then, the cantilever is reinforced by thickening the weak part with modes (g) and (h). Required conditions about parameters and gains are that, first, corresponding parameters fulfill the following inequalities, $a_{v 41}>0, a_{v 11}>0$, $a_{h 44}<0, a_{h 34}<0, a_{v 31}<0, a_{h 33}>0, a_{h 32}>0$, $a_{v 23}>0$, and second, priorities of the transformation modes should shift appropriately in each phases. For example, in order that priority of mode (f) and priority of mode (b) 

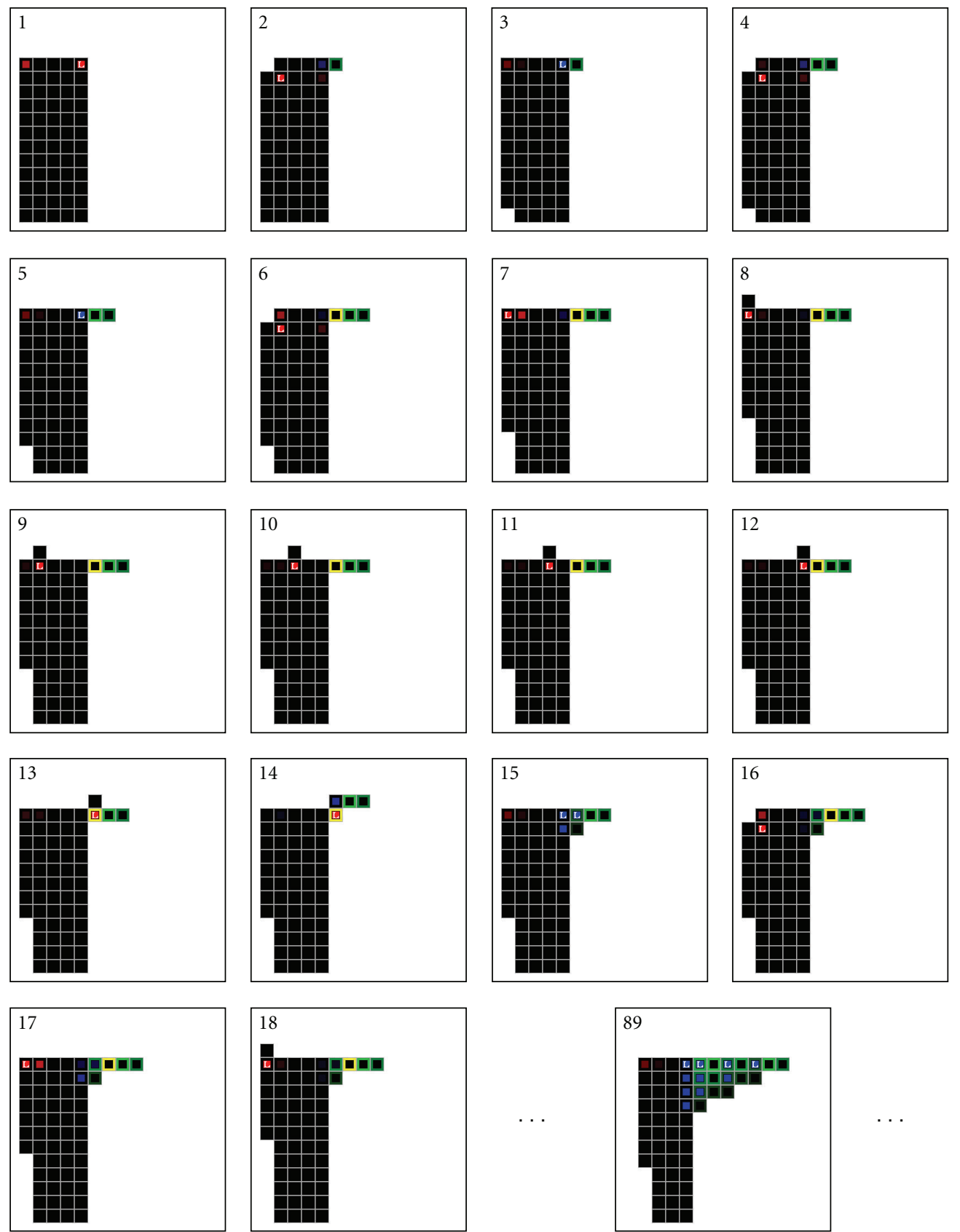

FIGURE 15: Simulation result of adaptive cantilever construction from $12 \times 5$ rectangle structure.

are switched in overloaded states, following two inequalities must be fulfilled, $\left|a_{v 11}\right|>\left|a_{h 33}\right|,\left|G^{+} a_{h 33}\right|>\left|a_{v 11}+a_{v 31}\right|$. Formula (5) shows parameters fulfilling all the conditions:

$$
\begin{aligned}
A_{v} & =\left[\begin{array}{cccc}
16 & 0 & 0 & 0 \\
0 & 0 & 18 & 0 \\
-2 & 0 & 0 & 0 \\
4 & 0 & 0 & 0
\end{array}\right], \quad A_{h}= \\
\mathrm{G}^{+} & =3, \quad \mathrm{G}^{-}=0.6 .
\end{aligned}
$$

To simulate the load adaptive transformations on a computer, it is required to estimate the output voltage $S$ $[\mathrm{mV}]$ of the strain gauge sensor circuit of the modules. In this paper, we use estimation formulas (6) and (7) derived from the result of load experiments using actual machines and linear beam theory:

$$
\begin{gathered}
S^{\prime}=40 n_{0}+\sum_{x=1}^{x_{\max }}(150 x-20) n_{x} \\
S=\left(\frac{1}{h^{2}}\right)\left(\frac{i}{h}\right) S^{\prime}
\end{gathered}
$$




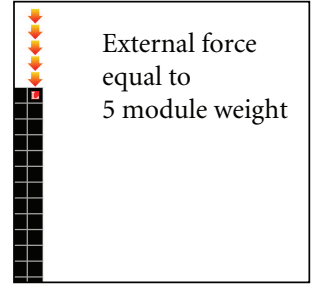

(a)

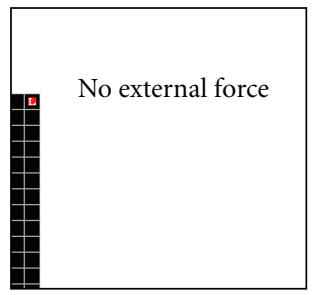

(e)

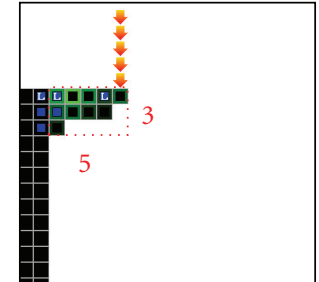

(b)

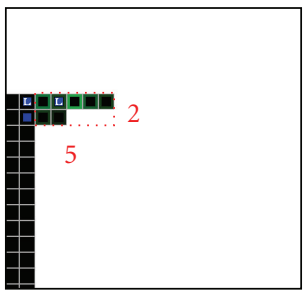

(f)

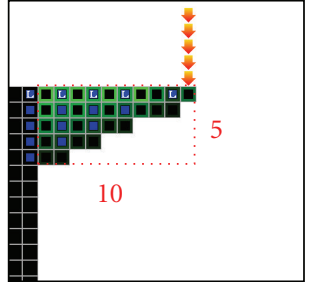

(c)

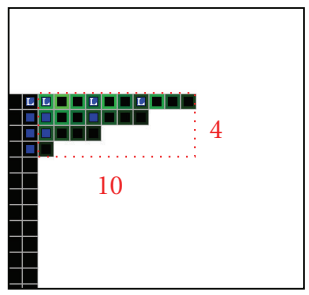

(g)

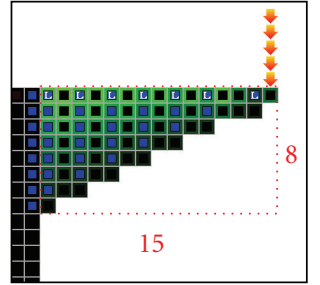

(d)

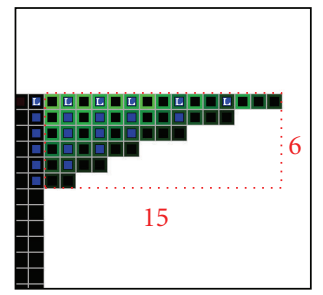

(h)

FIGURE 16: Difference of cantilever shapes caused by external force ( $(a-d)$ Simulation result of adaptive cantilever construction on a load condition caused by external force and weight of modules themselves, (e-h) on a load condition caused by weight of modules themselves only).

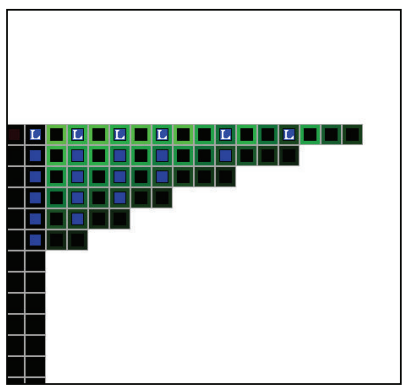

(a)

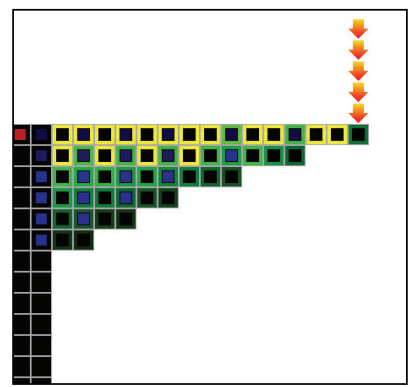

(b)

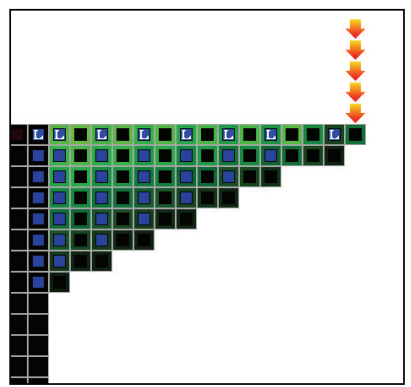

(c)

FiguRE 17: Reaction to additional external force in a process of bridge construction (simulation result of external force acting at the tip of the bridge).

Here, $x$ coordinate is directed toward right direction with the origin at the target module whose $S$ should be estimated, and $n_{x}$ is the number of modules on the $x$ th column (see Figure 14(a)). Thus, formula (6) means $S^{\prime}$ becomes larger as more modules exist far in the right direction from the target module. In formula (7), $h$ is the number of modules connecting to the left-neighboring modules on the column including the target module, and $i$ indicates what number the target module exist from the bottom in the column (see Figure 14(b)). Thus, formula (7) means, following to the beam theory, $S$ is inversely proportional to square of thickness of the beam and becomes smaller as the target module exists far from the top surface of the beam.

Simulation result of the transformation process generated by the criterion in formula (5) with setting threshold of the sensor output $S_{\text {threshold }}=400$ to be considered as overloaded is shown in Figure 15. In the figure, green color in outer parts of modules becomes bright in proportion to strain gauge outputs of the modules, and yellow color means that the strain is beyond threshold level. When an overloaded module occurs at the 6th step, bridge construction motion is temporarily stopped and load adaptive transformation is performed until the 15th step. Continuing this process, at the 89th step, the modules autonomously construct a bridge whose thickness becomes smaller from the root to the tip, which is an appropriate shape to support the bridge in the gravitational environment.

5.3. Discussion of Practicality. In this chapter, we discuss practicality of the proposed method based on the viewpoint of adaptability to more complicated load conditions including external force.

We show that the proposed algorithm is applicable to load conditions caused not only by weight of the modules but also by external force. Here, assuming that CHOBIE II conveys a cabin baggage, we demonstrate the motion of lengthening a cantilever structure with external force acting downward at the tip of the cantilever. Figures 16(a)$16(\mathrm{~d})$ show a simulation result in case the external force is equal to the weight of 5 modules. It is confirmed that the 
modules adaptively construct a cantilever, and the shape of the cantilever is about 1.35 times as thick as that constructed without the external force as shown in Figures 16(e)-16(h), compared in the same lengths (length of 5, 10, and 15 modules).

We also tried another load condition that external force is suddenly added after construction of a 15 module-length bridge (same shape as Figure 16(h)). The external force is equal to the weight of 5 modules. Simulated transformation process is shown in Figure 17. There is no overloaded module before loading the external force (Figure 17(a)). After loading, 16 modules in the structure become overloaded state (Figure 17(b)), and structural transformations are generated so as to dissolve all of the states (Figure 17(c)). The finally reinforced shape is the same as Figure 16(d), and then the modules begin to lengthen the bridge. This result suggests that, even if load condition suddenly changes during structural construction process, the algorithm enables the modules to switch the transformation mode to adapt to the load condition, and the whole shape converges in an appropriate one.

Although this demonstration is a simple load condition, it can be expected that the proposed method is also applicable to various load conditions.

\section{Conclusions}

In this paper, we introduced two new methods in order to enable CHOBIE II to construct a bridge-like structure with adapting to a load condition. The first method is for making and enlarging desirable local shapes. We confirmed that CHOBIE II can transform to characteristic configurations as a cantilever structure due to this method. The second method enables the modules to change their criterion for generating transformation so that the modules estimate more undesirably about shapes which cause overstressed states. Adopting these methods, we demonstrated a motion of constructing a cantilever with reinforcing stress-concentrated portions. In addition, we discussed a transformation process in a load condition including an external force. From the result, it can be expected that the proposed method is also applicable to various load conditions within a framework of a decentralized control method. The future works are to input this algorithm into actual modules and verify the availability of the proposed load adaptation algorithm.

\section{References}

[1] T. Fukuda, S. Nakagawa, Y. Kawauchi, and M. Buss, "Structure decision method for self organising robots based on cell structures-CEBOT," in Proceedings of the IEEE International Conference on Robotics and Automation (ICRA '89), vol. 2, pp. 698-700, Scottsdale, Ariz , USA, May 1989.

[2] D. Rus and M. Vona, "Self-reconfiguration planning with compressible unit modules," in Proceedings of the IEEE International Conference on Robotics and Automation (ICRA '99), pp. 2513-2520, May 1999.
[3] M. Yim, W. M. Shen, B. Salemi et al., "Modular selfreconfigurable robot systems," IEEE Robotics and Automation Magazine, vol. 14, no. 1, pp. 43-52, 2007.

[4] M. Yim, D. G. Duff, and K. D. Roufas, "PolyBot: a modular reconfigurable robot," in Proceedings of the IEEE International Conference on Robotics and Automation (ICRA '00), pp. 514520, April 2000.

[5] D. Rus and M. Vona, "Crystalline robots: self-reconfiguration with compressible unit modules," Autonomous Robots, vol. 10, no. 1, pp. 107-124, 2001.

[6] S. Murata, K. Kakomura, and H. Kurokawa, "Toward a scalable modular robotic system," IEEE Robotics and Automation Magazine, vol. 14, no. 4, pp. 56-63, 2007.

[7] W. M. Shen, B. Salemi, and P. Will, "Hormone-inspired adaptive communication and distributed control for CONRO self-reconfigurable robots," IEEE Transactions on Robotics and Automation, vol. 18, no. 5, pp. 700-712, 2002.

[8] D. J. Christensen, E. H. Ostergaard, and H. H. Lund, "Metamodule control for the ATRON self-reconfigurable robotic system," in Proceedings of the 8th International Conference on Intelligent Autonomous Systems (IAS '8), pp. 685-692, 2004.

[9] J. Kubica, A. Casal, and T. Hogg, "Complex behaviors from local rules in modular self-reconfigurable robots," in Proceedings of the IEEE International Conference on Robotics and Automation (ICRA '01), vol. 1, pp. 360-367, 2001.

[10] M. Shimizu, A. Ishiguro, and T. Kawakatsu, "Slimebot: a modular robot that exploits emergent phenomena," in Proceedings of the IEEE International Conference on Robotics and Automation (ICRA '05), pp. 2982-2987, April 2005.

[11] K. Gilpin, K. Koyanagi, and D. Rus, "Making self-disassembling objects with multiple components in the robot pebbles system," in Proceedings of the IEEE International Conference on Robotics and Automation (ICRA '11), pp. 36143621, Shanghai, China, May 2011.

[12] P. Bhat, J. Kuffner, S. Goldstein, and S. Srinivasa, "Hierarchical motion planning for self-reconfigurable modular robots," in Proceedings of the IEEE/RSJ International Conference on Intelligent Robots and Systems (IROS '06), pp. 886-891, October 2006.

[13] H. Bojinov, A. Casal, and T. Hogg, "Emergent structures in modular self-reconfigurable robots," in Proceedings of the IEEE International Conference on Robotics and Automation (ICRA '00), pp. 1734-1741, April 2000.

[14] M. Koseki, K. Minami, and N. Inou, "Cellular robots forming a mechanical structure (evaluation of structural formation and hardware design of "CHOBIE II")," in Proceedings of the International Symposium on Distributed Autonomous Robotic Systems (DARS '04), pp. 131-140, 2004.

[15] Y. Suzuki, N. Inou, M. Koseki, and H. Kimura, "Reconfigurable modular robots adaptively transforming a mechanical structure (numerical expression of transformation criteria of "CHOBIE II" and motion experiments)," in Distributed Autonomous Robotic Systems 8, H. Asama, H. Kurokawa, J. Ota, and K. Sekiyama, Eds., pp. 393-403, Springer, New York, NY, USA, 2009.

[16] Y. Suzuki, N. Inou, H. Kimura, and M. Koseki, "Reconfigurable group robots adaptively transforming a mechanical structure (extended criteria for load-adaptive transformations)," in Proceedings of the IEEE/RSJ International Conference on Intelligent Robots and Systems (IROS '08), pp. 877-882, September 2008. 
[17] H. M. Frost, The Laws of Bone Structure, C. C. Thomas, 1964.

[18] Y. C. Fung, N. Perrone, and M. Anliker, Biomechanics: Its Foundations and Objectives, Prentice Hall, New York, NY, USA, 1972.

[19] S. C. Cowin and D. H. Hegedus, "Bone remodeling I: theory of adaptive elasticity," Journal of Elasticity, vol. 6, no. 3, pp. 313326, 1976.

[20] R. Huiskes, H. Weinans, H. J. Grootenboer, M. Dalstra, B. Fudala, and T. J. Slooff, "Adaptive bone-remodeling theory applied to prosthetic-design analysis," Journal of Biomechanics, vol. 20, no. 11-12, pp. 1135-1150, 1987. 

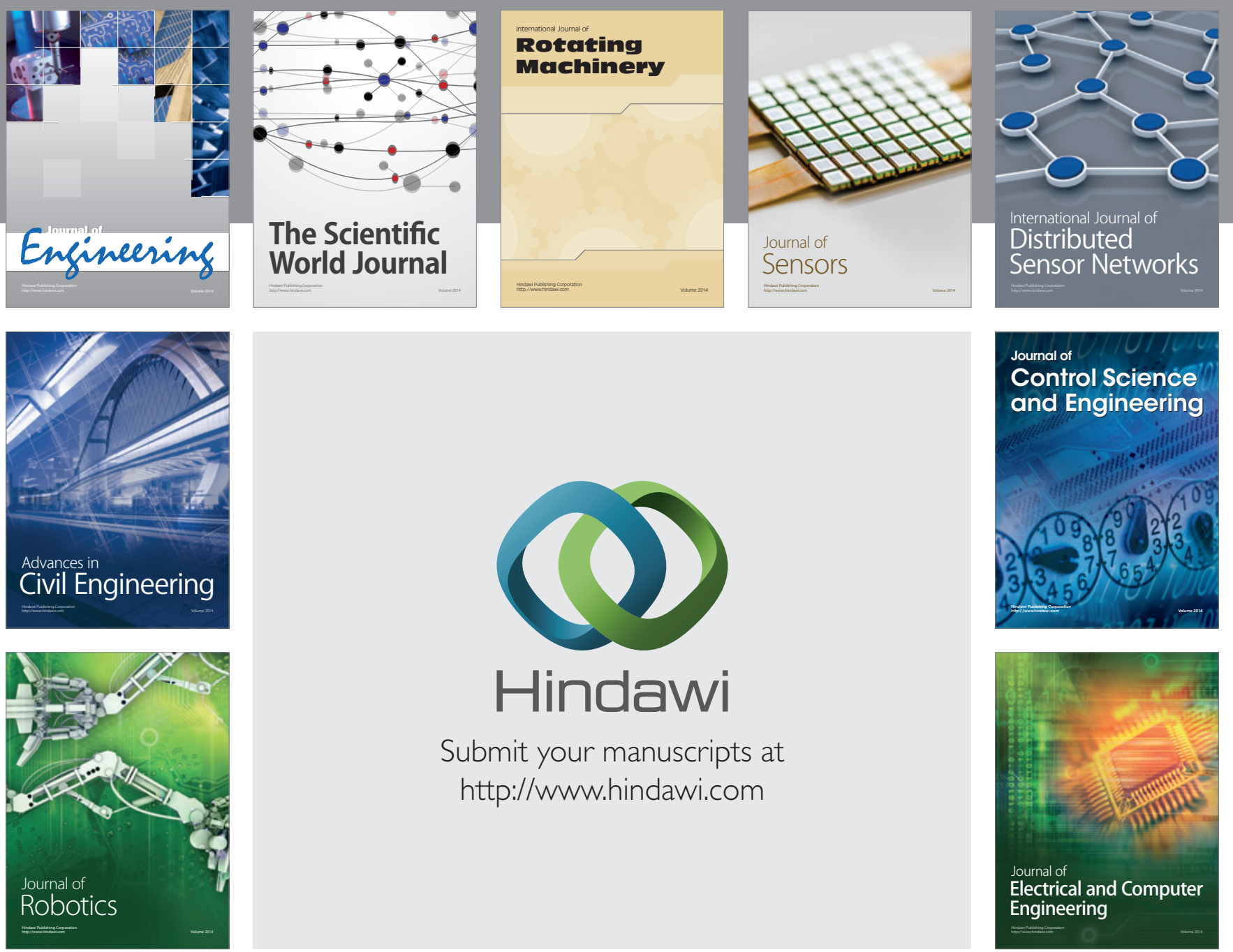

Submit your manuscripts at

http://www.hindawi.com
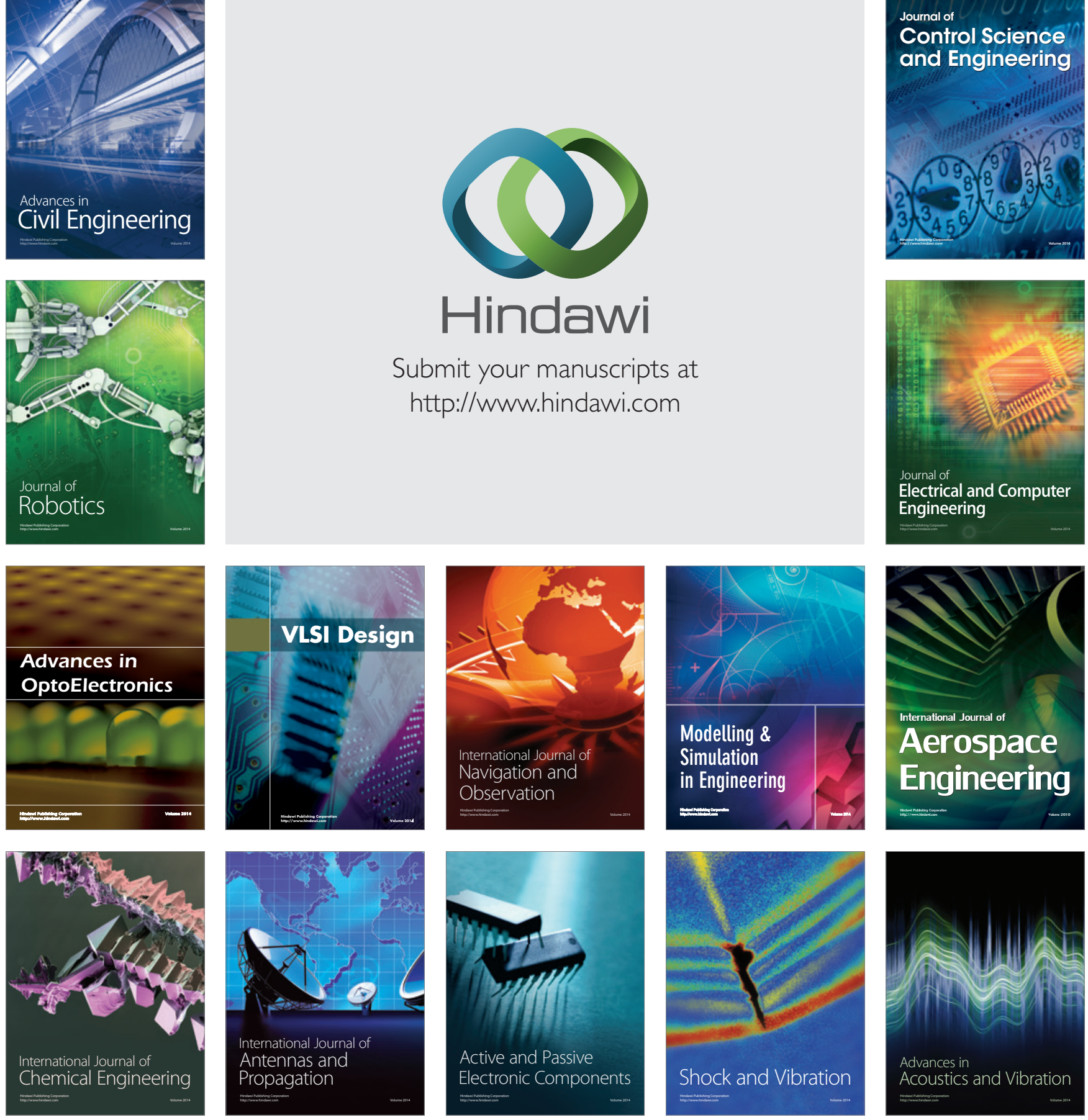